\title{
Extraperitoneal Approach During Peritonectomy in the Right Upper Quadrant for Peritoneal Metastases from Ovarian Malignancies
}

\author{
MIKLOS ACS ${ }^{1}$, HUBERT LEEBMANN $^{1}$, SEBASTIAN HÄUSLER $^{2}$, PHILIPP HARTER $^{3}$ and POMPILIU PISO ${ }^{1}$ \\ ${ }^{1}$ Department of General and Visceral Surgery, Hospital Barmherzige Brüder, Regensburg, Germany; \\ ${ }^{2}$ Department of Gynecology and Gynecologic Oncology, \\ Hospital Barmherzige Brüder, Clinic St. Hedwig, Regensburg, Germany; \\ ${ }^{3}$ Department of Gynecology and Gynecologic Oncology, Kliniken Essen-Mitte, Essen, Germany
}

\begin{abstract}
Aim: To present the extraperitoneal approach for the removal of peritoneal metastases in the right upper abdomen in patients with ovarian cancer and to evaluate safety and potential advantages with comparison with the traditional approach. Patients and Methods: Detailed description of the right upper quadrant peritonectomy as extraperitoneal approach. Procedure-specific short-term complications were retrospectively analyzed in a cohort of patients. Results: Sixty-four patients were included. Fullthickness diaphragmatic resection was performed in $17 \%$ of primary cases, and in $44 \%$ of the patients with recurrent ovarian carcinoma. The rate of complete cytoreduction (CC0) was $70 \%$. The most common postoperative complication was pleural effusion (32\%). Conclusion: The extraperitoneal approach for peritonectomy of the right upper quadrant in patients with ovarian cancer is feasible, with improved access to the right subdiaphragmatic area. This enables a high rate of complete cytoreduction, and simplified and safe surgical dissection in an uncontaminated area under secured vascular structures. The early postoperative outcomes are comparable to those of the traditional transperitoneal approach.
\end{abstract}

In industrialized countries, ovarian cancer is the leading cause of gynecological cancer-related mortality. The poor

This article is freely accessible online.

Correspondence to: Miklos Acs, Department of General and Visceral Surgery, Krankenhaus Barmherzige Brüder, Prüfeninger Strasse 86, 93049 Regensburg, Germany. Tel: +49 94136994400, Fax: +49 9413692206,e-mail: miklos.acs@barmherzige-regensburg.de

Key Words: Extraperitoneal peritonectomy, cytoreductive surgery, ovarian cancer, upper abdomen. prognosis is attributed to the fact that two-thirds of patients have advanced-stage ovarian cancer at diagnosis (1). The majority of these women have extensive intra-abdominal disease with significant peritoneal involvement at the time of diagnosis, resulting in low overall cure rates. The most important prognostic factor remains the residual disease status after cytoreductive surgery (CRS) (2-4).

In advanced-stage ovarian cancer, up to $40 \%$ of patients present with bulky metastases in the diaphragmatic peritoneal region, mainly on the right side (5-7). Metastases appear in this region as a consequence of transcoelomic tumorous implants from the floating malignant cells in the peritoneal fluid, which circulates clockwise in the peritoneal cavity (8).

Maximal effort to achieve complete cytoreduction with the resection of diaphragmatic metastases is associated with improved survival $(5,9,10)$. For this reason, gynecological oncologists commonly perform upper abdominal surgery (9, 11). Not only in the primary but also in the recurrent setting, complete cytoreduction seems to deliver survival benefit. Recently results from the DESKTOP III trial, presented at the American Society of Clinical Oncology meeting in Chicago, highlighted a survival benefit in favor of secondary complete CRS in comparison to chemotherapy alone (12).

Diaphragmatic peritonectomy and full-thickness resection constitute an effective way to remove diaphragmatic carcinomatosis and achieve complete cytoreduction. These interventions frequently result in intrathoracic and pulmonary complications, although long-term morbidity is uncommon. Diaphragmatic surgery is mostly limited to stripping. However, if the tumor infiltrates the muscle fibers of the diaphragm, full-thickness resection is necessary, and the opening of the pleural cavity is inevitable.

After having reviewed the literature on diaphragmatic surgery for ovarian cancer, the authors found that descriptions of surgical techniques predominantly discuss the 
transperitoneal approach. Nevertheless, upper abdominal peritonectomy from extraperitoneal approach constitutes a technique safely applied in surgical oncology that may offer some advantages. The significance of this issue is given by the analysis of the Gynecologic Oncology Group 182 trial, which showed that the diaphragm is the most common localization for residual tumor after surgery in advanced ovarian cancer (13).

The primary aim of this article was to describe the surgical technique of right subdiaphragmatic peritonectomy through extraperitoneal access, which is used by surgical oncologists for peritoneal surface malignancies, and to evaluate its safety. Additionally, we compared this approach to the traditional peritonectomy procedure performed by gynecological oncologists at most oncology centers. Furthermore, another objective of this report was to critically discuss the most relevant and controversial aspects of the two cytoreductive surgical techniques, outlining the possible benefits of the extraperitoneal surgical technique. Indeed, there are some differences between the traditional transperitoneal approach and the extraperitoneal dissection technique as described by the Peritoneal Surface Oncology Group International $(14,15)$.

In addition, the Authors conducted a retrospective study to evaluate the typical, diaphragmatic surgery-related early postoperative complications following peritoneal stripping and full-thickness resection with extraperitoneal approach in patients with ovarian cancer. Our work may provide an opportunity to expand the armamentarium of the surgical oncologists and gynecological oncologists to achieve a higher rate of complete tumor reduction

\section{Patients and Methods}

Data were assessed of 146 consecutive patients treated with CRS (with or without subsequent hyperthermic intraperitoneal chemotherapy, HIPEC) during January $1^{\text {st }} 2011$ to April $30^{\text {th }} 2020$ for primary or recurrent invasive ovarian or fallopian tube and primary peritoneal cancer at the Barmherzige Brüder Regensburg Hospital, Germany, a tertiary referral center certified for treatment of peritoneal surface malignancies. The indications for CRS and HIPEC were recommended as an individual therapy by an interdisciplinary team in the setting of a tumor conference, supported by the patient's preferences and the referring oncologist. Ninety-nine of the 146 patients with ovarian cancer underwent CRS with HIPEC, whereas 47 underwent CRS only. Of the 146 patients who were treated within the study period, we included only those who underwent peritonectomy and full-thickness resection of the right upper diaphragm through the extraperitoneal approach with or without liver capsule resection: 55 in the CRS plus HIPEC group and nine in the CRS-only group. Thus, altogether 64 patients were included in the present study. Documented data of each patient were reviewed, and surgical, pathological, and Intensive Care Unit records were extracted. The operations were performed by an ovarian team consisting of a gynecological oncologist and a surgical oncologist.
Operative and patient data were entered into the German national HIPEC registry, initiated by the German Society for General and Visceral Surgery, and retrospectively analyzed for this study. The patients agreed to data entry before their initial surgery by written and oral informed consent. The choice of treatment for each patient was determined by a multidisciplinary team.

The completeness of cytoreduction (CC) was scored as proposed by Sugarbaker: CC-0: No residual disease; $\mathrm{CC}-1$ : residual nodules measuring less than $2.5 \mathrm{~mm}$; CC-2: residual nodules measuring between $2.5 \mathrm{~mm}$ and $2.5 \mathrm{~cm}$; and CC-3: residual nodules greater than $2.5 \mathrm{~cm}$ (16). However, this scoring system cannot be compared to the "greatest dimension of single largest residual lesion" following CRS, which constitutes an accepted measurement in the field of gynecological oncology $(17,18)$. Nevertheless, both systems are completely identical if there is no macroscopic residual disease at the end of the CRS.

Description of the surgical techniques. Patient positioning is similar for both procedures: Modified lithotomy position with the gluteal fold at the end of the operating table, allowing full access to the whole abdomen and peritoneal surfaces. In both techniques, the abdomen is opened through a midline incision, extending from the xiphoid above to the pubis below, to gain maximal access to all abdominal compartments.

Traditional transperitoneal approach with liver mobilization. According to the traditional transperitoneal approach, the procedure for which is described in detail elsewhere $(5,19,20)$, the first steps are the mobilization and then medialization of the liver for better exposure. The falciform ligament, containing the round ligament of the liver (ligamentum teres hepatis), is divided and ligated. Peritoneal dissection of the right anterior coronary ligament starting medially towards lateral comes next after identification of the inferior vena cava, followed by the separation of the right triangular ligament. The dissection is continued up to the bifurcation of the right coronary ligament, where both the anterior and the posterior right coronary ligaments are divided, thus allowing medialization of the right lobe of the liver. Mobilization is continued until the nude area of the liver appears. Dissection is sometimes performed in the lateromedial direction, first separating the triangular ligament from the diaphragm. After the liver is mobilized and the borders of the planned resection line are demarcated with clamps, cranially on the anterior edge of the right hemidiaphragm along the costal margin and caudally from Gerota's fascia, peritonectomy follows. This can be performed with bipolar scissors (21). If diaphragmatic resection is necessary, it is carried out by a stapling device either without opening of the pleural cavity in case of shallow infiltration or through the pleural cavity opening for full-thickness resection in the case of deeply invading tumor. Lastly, the generated defect is closed by interrupted horizontal mattress or by figure-of-eight permanent sutures. The aim of liver mobilization prior to the peritonectomy is to ensure full exposure of diaphragmatic disease and to access the bare area of the liver (22).

Extraperitoneal approach. The extraperitoneal approach was originally described by Sugarbaker and the Peritoneal Surface Oncology Group International as a peritonectomy procedure (15, 23-25) and is practiced by surgical oncologists in cases of peritoneal carcinosis from various malignancies such as cancer of colorectal or gastric origin, pseudomyxoma peritonei, and mesothelioma. The 


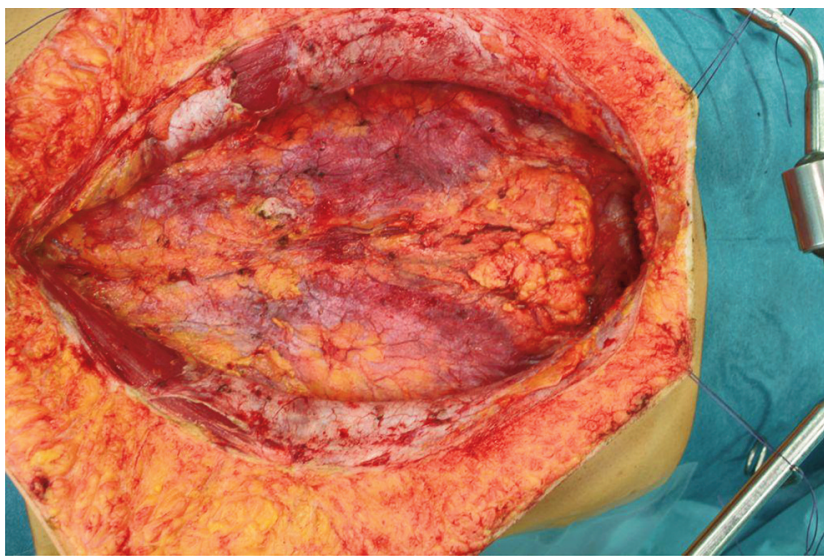

Figure 1. Circular exposure of the peritoneal sac by extraperitoneal dissection. The skin with holding sutures on the retractor is stretched out, which provides extended exposure of the surgical site.

device used for peritonectomy and preparation is a $3 \mathrm{~mm}$ ball-tipped electrosurgical handpiece used on pure cut at high voltage. Tissue damage due to high voltage can be prevented by rapid cutting movements and regular irrigation. Xyphoidectomy for better exposure of the right or the left hemiabdomen is rarely necessary. Peritonectomy in the right hemiabdomen is facilitated by a selfretraining retractor. In this case the edges of the skin can be stretched out by holding sutures bound to the retractor, providing better exposure of the surgical site (Figure 1). Preparation and peritonectomy in the right hemiabdomen are implemented through electrocoagulation and not through blunt preparation in order to prevent bleeding from the several vascular connections between the diaphragm and the peritoneum. After the fascia has been dissected, the dissection is continued strictly extraperitoneally for about 5-10 $\mathrm{cm}$ in the anterolateral direction while the peritoneal sac remains closed and intact. In the upper abdomen, the peritonectomy is extended several centimeters towards the subdiaphragmatic areas on both sides. The preparation immediately below the costal arch enables a self-retaining retractor to be used. The peritoneum can then be opened through a short incision. Especially in previously operated patients, its line should be chosen paramedian outside of the old scar to avoid serosal lesions or transmural defects of the intestine. This peritoneal window can be used for an orientational exploration of the abdominal cavity and the internal organs, thus excluding contraindications to surgery, particularly multilocular small intestinal involvement. The entrance to the appropriate preparation layer subxyphoideally and subdiaphragmatically lies at the area of the fatty triangle. Preperitoneal fat and peritoneum can be easily separated from the diaphragm here. The subphrenic dissection continues until the junction of the hepatic veins into the vena cava are encountered.

The confluence of the hepatic veins with the vena cava is usually easily palpable at the indentation between the right and middle hepatic veins. During extraperitoneal dissection, this area can usually be palpated long before the vena cava becomes visible. At this point, careful preparation is necessary in order not to injure the major vessels. Injury can be avoided by frequent palpation of the recess between the right and medial hepatic vein, the junction of which to the retrohepatic segment of the inferior vena cava (IVC)

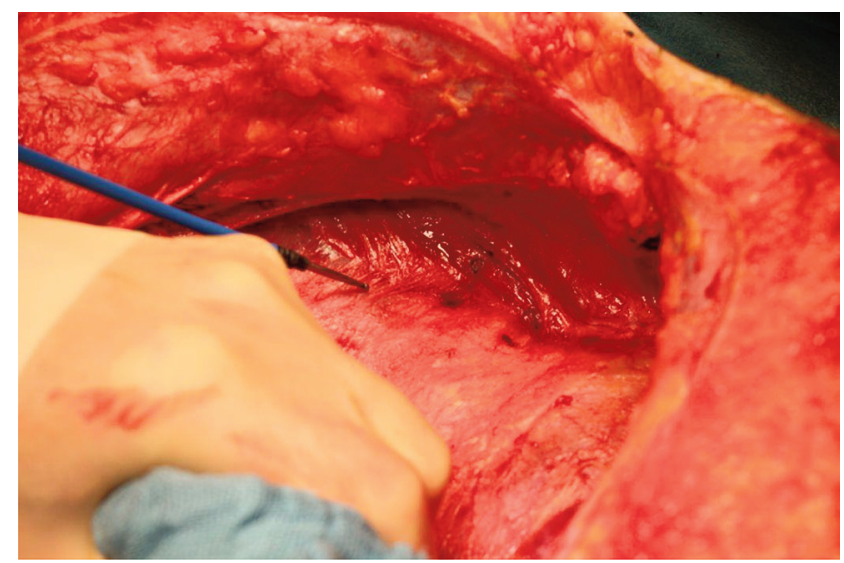

Figure 2. Extraperitoneal peritonectomy through electrocoagulation in the right subphrenic region.

constitutes the cranio-medial border during this procedure. The preparation in the area of the central tendon is technically demanding because the anatomical layers between the diaphragm and the peritoneum are extremely flimsy here. Next, the separation of the peritoneum in the right upper quadrant is initiated from the posterior rectus sheath. Strong traction needs to be applied on the peritoneum while moving forward with stripping with electrocautery, thus bringing the diaphragmatic muscle into view and medializing the liver at the same time (Figure 2). Contratraction is achieved by pulling upwards of the right costal margin by a self-retaining retractor. Several small vessels draining from the diaphragm musculature to the peritoneum should be coagulated simultaneously with stripping to reduce blood loss.

The stripping of the tumorous right hemidiaphragmatic peritoneum is continued until the bare area of the liver is encountered. Here the peritoneum bends over into Glisson's capsule of the liver, which can be stripped en bloc from the surface of the liver if tumorous implants are present. During the dissection in this area, the right, medial and left hepatic veins draining into the anterior side of the IVC will be encountered. The right phrenic nerve passes through the vena caval hiatus laterally in the diaphragm at the level of the eighth thoracic vertebra and divides just superior to or at the level of the diaphragm. It is generally not encountered during peritonectomy until the bare area of the liver is exposed. If the tumor is in close proximity to vascular structures, the hepatic veins must be carefully examined before subphrenic peritonectomy is carried out. Experience shows that tumorous infiltration of these rarely occurs, as their position remains retroperitoneal. The right subdiaphragmatic peritoneum, the capsule covering the surface of the liver and the right subhepatic space covered by the peritoneum (Morison's pouch) constitute an envelope. The surgery aims to remove it en bloc, in a manner continuing the subhepatic peritonectomy in cranial direction, while moving on with the subdiaphragmatic peritonectomy in caudolateral direction. The right dorsal border of the preparation comprises Gerota's fascia and the perirenal fat covering the right kidney. At this point, the right adrenal gland is visualized. It must be protected from any injuries while peeling off the tissues in the subhepatic region. As the peritoneal reflection at the posterior aspect 


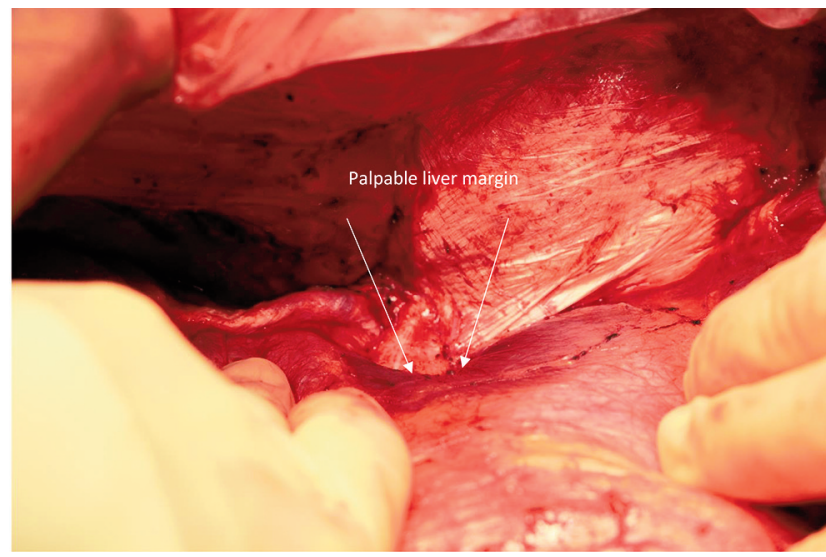

Figure 3. After peritonectomy, the right and medial hepatic veins draining into the anterior surface of the inferior vena cava can be visualized.

of the liver is stripped (posterior right coronary ligament), the vena cava and the veins of the caudate lobe running between the first liver segment and the vena cava must be protected from injury.

The falciform ligament is dissected off the liver capsule and remains attached to the tumorous peritoneum unless Glissonectomy is also required. In the latter situation, these structures can be resected en bloc. When preparation is completed, the anterior branches of the phrenic vein and artery can be visualized, together with the right hepatic vein and the vena cava running under it, in connection with the smaller retrohepatic veins (Figure 3 and Figure 4). Branches of the right phrenic artery and vein can be divided if necessary during the procedure of the peritonectomy.

If the tumor is tightly adherent to or infiltrating into the diaphragmatic fibers, especially at the central tendon, the tumor must be removed with full-thickness resection. Full-thickness resection can often be performed by an elliptical incision with bipolar scissors or electrocautery and closed by interrupted sutures. Another option includes exerting traction by a Kelly clamp on the tumor-infiltrated diaphragmatic muscle section and then resecting with thoracoabdominal stapling device and finally closing the diaphragm in a single step. In this case, the pleural cavity is not opened. The latter method is performed in a similar manner by oncological surgeons and gynecological oncologists.

Intra- and postoperative results. Patients in the first study group (55/64; 86\%), following CRS which was achieved by parietal and visceral peritonectomy procedures, received bidirectional HIPEC with closed-abdomen technique. Cisplatin at $75 \mathrm{mg} / \mathrm{m}^{2}$ and doxorubicin at $15 \mathrm{mg} / \mathrm{m}^{2}$ body surface area were administered at $42^{\circ} \mathrm{C}$ for 60 or $90 \mathrm{~min}$. Considering the small number of patients in the CRS-only group $(n=9)$, at the evaluation of the postoperative outcome, the two study groups, i.e., all 64 patients, were analyzed in one block.

Procedure-specific postoperative short-term complications were registered according to the description of the operation, anesthesiology, and Intensive Care Unit records, as well as of ward documentation. As stipulated in the hospital protocol, postoperative chest X-ray examination was performed in all patients undergoing operation, regardless of symptoms. At our Institution, intraoperative

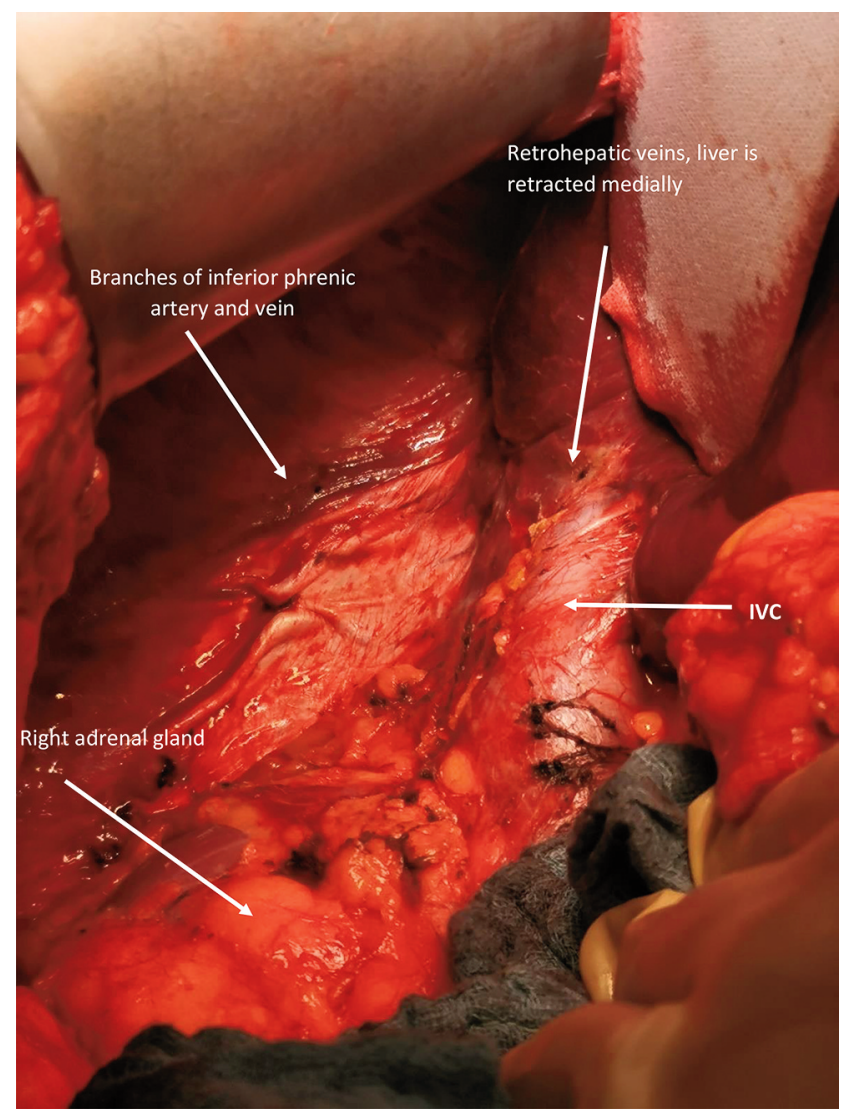

Figure 4. Completed peritonectomy in the right upper quadrant. The liver has been mobilized and retracted medially. The branches of the inferior phrenic artery and vein, the inferior vena cava (IVC) with the retrohepatic veins and the right adrenal gland visualized.

chest tube placement was applied routinely in the following situations: If during full-thickness resection or unwarily the thoracic cavity was opened; during peritoneal stripping; or if it was considered indispensable by the operating surgeon in cases of extensive diaphragmatic intervention. In addition, chest drainage was also inserted immediately after postoperative HIPEC treatment when a fluid discrepancy emerged between the total inflow and the outflow, with the accumulation of the unaccounted fluid in the thoracic cavity confirmed by ultrasonography.

\section{Results}

Overall, $72 \%$ of the 64 patients who underwent peritonectomy and full-thickness resection of the right upper diaphragm, with or without liver capsule resection, received primary CRS. Patients in the primary setting had International Federation of Obstetrics and Gynecology stage IIIC-IV disease. The remaining $28 \%$ underwent secondary or tertiary CRS (15\% and $13 \%$, respectively). Full-thickness diaphragmatic resection was necessary in $44 \%$ of the patients 
Table I. Procedures, surgical outcomes, and histological subtypes.

\begin{tabular}{lc}
\hline & Frequency, n (\%) \\
\hline Surgical procedures & \\
Peritonectomy in the right upper quadrant & $64(100 \%)$ \\
Full-thickness resection & $16(25 \%)$ \\
Primary & $8(12.5 \%)$ \\
Recurrent & $8(12.5 \%)$ \\
Liver capsule resection & $12(18 \%)$ \\
Intraoperative chest tube insertion & $34(53 \%)$ \\
Complete cytoreduction (no residual tumor) & $45(70 \%)$ \\
Residual disease $<1$ cm & $19(30 \%)$ \\
(not diaphragm-related residual disease) & \\
Histology & \\
High-grade serous ovarian, fallopian tube, & $40(62.5 \%)$ \\
and peritoneal cancer & \\
$1^{\text {st }}$ Recurrence & $8(12.5 \%)$ \\
$2^{\text {nd }}$ Recurrence & $7(11 \%)$ \\
Mucinous & $2(3 \%)$ \\
$2^{\text {nd }}$ Recurrence & $1(1.6 \%)$ \\
Transitional cell cancer & $1(1.6 \%)$ \\
$1^{\text {st }}$ Recurrence & $1(1.6 \%)$ \\
Yolk-sac tumor & $1(1.6 \%)$ \\
Granulosa cell tumor $1^{\text {st }}$ recurrence & $1(1.6 \%)$ \\
Immature teratoma & $1(1.6 \%)$ \\
Endometrioid & $1(1.6 \%)$ \\
Tumor & \\
Primary & $46(72 \%)$ \\
Recurrent & $18(28 \%)$ \\
Total & $64(100 \%)$ \\
\hline
\end{tabular}

with recurrent ovarian carcinoma and in $17 \%$ of patients in the primary setting. Full-thickness resection was carried out in 16 patients $(25 \%)$, while liver capsule resection was performed in $12(18 \%)$.

Procedures, surgical outcomes, and histological subtypes are listed in Table I.

The tumorous involvement of the right upper quadrant was completely resected in all patients. Furthermore, we analyzed each case in which the surgery failed to achieve macroscopic complete resection $(n=19 ; 30 \%)$ and found that the underlying cause for this was the disseminated fine nodular tumorous involvement of the small intestine and its mesentery. Two patients had tumor residues at other locations apart from the fine nodular involvement of the small intestine. In one of these patients, residual tumorous tissue remained interiliacally and was tightly adherent to the external iliac artery and vein. The other patient harbored 2 mm-sized disseminated metastases on the splenic capsule. Considering the advanced age of this patient, we resigned from multi-visceral resection to prevent increased morbidity related to splenectomy. Thus, cytoreduction with residual disease $(<1 \mathrm{~cm})$ was achieved by the end of the surgery for the whole study population.
Complications. Postoperative diaphragmatic surgery-related short-term complications occurred in 30 patients (47\%). The most common complication was pleural effusion, which developed in 21 out of 64 patients $(32 \%)$ in the postoperative period. Postoperative chest tube insertion was necessary in five patients $(8 \%)$, whereas thoracocentesis was performed in 10 patients (15\%). Additionally, in six cases (9\%), the pleural effusion was resolved by pharmacological treatment. Intraoperative chest tube insertion was carried out in 34 cases (53\%). Fluid discrepancy between the total inflow and the outflow after HIPEC occurred in five cases in our patient group $(8 \%)$. The accumulation of the unaccounted fluid in the thoracic cavity was confirmed with ultrasonography and was followed by chest tube insertion. Altogether, two consecutive diaphragm-related complications occurred in five patients and three similar complications in two more patients. Major complications of Clavien-Dindo grade III and IV occurred in $34 \%(n=22)$ and $3 \%(n=2)$ of patients, respectively.

In one patient $(1.5 \%)$, an infected suprahepatic seroma had developed, which was treated with ultrasonography-guided puncture. Subphrenic abscess was observed in one case (1.5\%), requiring computed tomography-guided insertion of an abdominal drain. Subcapsular liver hematoma occurred in one patient $(1.5 \%)$ and was treated successfully with ultrasonography-guided drain insertion. In one patient, the postoperative course was complicated by pleural effusion, which was managed by thoracocentesis; subsequently pneumothorax arose and thoracic drainage was applied. In the further course, a septate pleural empyema II $^{\circ}$ evolved, which required surgical intervention with thoracoscopic decortication and the removal of the pleural fluid. Each of the abovementioned complications was treated successfully with radiological or endoscopic interventions, and surgery in one case. Apart from this, two patients developed postoperative respiratory failure, requiring admission to the Intensive Care Unit and mechanical ventilation. In one of them, it occurred in the context of a septic disease resulting from the perforation of a duodenal ulcer. Further respiratory complications included pneumothorax $(n=3 ; 4.6 \%)$ requiring chest tube insertion, and pneumonia $(n=5 ; 8 \%)$, which was treated with antibiotics and respiratory therapy. Pulmonary embolism occurred in four patients $(6 \%)$, all of whom received pharmacological treatment.

Concerning the mortality rate, in our cohort, the 30-day postoperative mortality rate was $3 \%$ (two patients) considering the 64 patients. One of the two patients died on the $12^{\text {th }}$ postoperative day in the Intensive Care Unit due to sepsis and multiple organ failure due to leakage from jejunojejunostomy anastomosis. The second patient died in the surgical ward due to a sudden onset of ventricular fibrillation. None of the deaths appear to have been directly related to the diaphragmatic surgery but rather to the consequences of multiorgan resection performed during $\mathrm{CRS}$. 
Table II. Comparison of the transperitoneal and extraperitoneal approach in surgery.

\begin{tabular}{lc}
\hline Transperitoneal approach & Extraperitoneal approach \\
\hline $\begin{array}{l}\text { Opening of the peritoneal cavity } \\
\text { Initial liver mobilisation }\end{array}$ & $\begin{array}{c}\text { Considers embryology and anatomical layers } \\
\text { Thitiation of the parietal peritonectomy from posterior } \\
\text { to anterior and from caudal to cranial directions } \\
\text { Complete extraperitoneal centripetal dissection }\end{array}$ \\
$\begin{array}{l}\text { Dissection technically more demanding } \\
\text { Clamps are necessary for the dissection }\end{array}$ & $\begin{array}{c}\text { Dissection simplified, no contamination with ascites or tumorous deposits occurs } \\
\text { Dissection technically easier, facilitated by traction and contra-traction } \\
\text { Short learning curve }\end{array}$ \\
$\begin{array}{l}\text { Glissonectomy only possible as separate specimen } \\
\text { Enables en bloc resection of the liver capsule }\end{array}$
\end{tabular}

\section{Discussion}

Since the seminal publications of Hudson and Chir in 1968 and 1973, respectively, describing the technique of "radical oophorectomy," consensus exists on en-bloc visceral tumor resection from retroperitoneal access in pelvic CRS for ovarian cancer $(26,27)$.

After the description of the two distinct methods, the conventional approach described by Montz et al. in 1989 (28), and the extraperitoneal approach described by Sugarbaker in 1995 (23), several decades later, gynecological oncologists and oncologic surgeons still continue to perform subdiaphragmatic peritonectomy in a different manner. The complex anatomy of the right upper abdomen and the dissemination of metastatic tumor nodes in close proximity to the vital structures makes peritonectomy in this area the most technically demanding part of CRS.

The extraperitoneal approach presented in this report might offer several benefits as compared to the traditional transperitoneal approach in right diaphragmatic surgery. The main features of the two different approaches are listed in Table II. In the extraperitoneal approach, liver mobilization and peritonectomy occur simultaneously, thus, mobilization of the liver prior to peritonectomy is not necessary in contrast to two-step traditional peritonectomy. Another advantage of extraperitoneal peritonectomy is that transperitoneal adhesiolysis can be avoided. Therefore, the upper abdominal peritoneum and the liver capsule can be removed as a single specimen while the important vascular structures can safely be visualized and palpated.

However, the most remarkable difference between the two operative techniques is most prominent when the tumorous disease is inseparable from Glisson's capsule. In the transperitoneal approach, the two sides of the tumorous plate (consisting of the peritoneum and the liver capsule) are separated, unnecessarily encumbering the resection of the tumorous layer in two steps. Since orientation in the tumorous environment is extremely difficult, in such cases, as described by Pathiraja et al. (22), the main vascular structures must be identified cranially, starting from the hilum of the liver. In contrast, in the extraperitoneal approach, cutting through the tumorous tissue can be avoided because preparation during the entire intervention respects the embryological anatomical layers under constant visualization of the essential vascular structures. In this case, the dissection continues along the superior part of the IVC until the posterior wall of the right hepatic vein is encountered.

The apparent correlation between optimal cytoreduction and improved survival in patients with with peritoneal metastases of ovarian cancer above the pelvis has resulted in an increasing rate of upper abdominal oncological surgery at many centers (29). Nevertheless, the addition of diaphragmatic procedures increases the incidence of postoperative respiratory complications. Direct comparison with other reports and evaluation of the postoperative morbidity rates is encumbered by the fact that simultaneously with diaphragmatic surgery, an additional radical intervention is performed in the abdominal cavity and the protocol for insertion of intraoperative chest tube might be different at each center. Thus, proper analysis of the underlying causes of complications becomes difficult. The actual outcome data may also be distorted by the difference in the indications for chest radiography, whether it is performed for all patients or only those who develop respiratory symptoms. During the course of the present study, all 64 patients underwent postoperative thorax radiography. On the other hand, 34 patients $(53 \%)$ were subjected to intraoperative chest tube insertion, so it was not possible to assess the true occurrence of intrathoracic complications without bias. The rate of full-thickness resection in our study, as described above, was $25 \%$ (16 patients). Several authors give different rates for diaphragmatic resection. For instance, Cliby et al. (7) from the Mayo Clinic suggested that diaphragmatic resection was performed in roughly $10 \%$ of the cases, whereas Chi et al. (30) from Memorial Sloan Kettering Cancer Center reported a rate of $14 \%$. Chèreau et al. (31) from Paris referred a rate of $15 \%$, while that of Ye et al. (32) from the Shanghai Cancer Center was $17.3 \%$ and Zapardiel et al. (33) study group from Milan reported a rate of full-thickness resection of $29.5 \%$. The rather high rate of full-thickness 
resection in our study might be attributed to the relatively high proportion of patients with recurrent ovarian cancer $(28 \%)$, who were more likely to require full-thickness resection. The high frequency of an infiltrating diaphragmatic involvement in recurrent disease suggests that surgical resection might have been incomplete in the primary setting. A report from the Mayo Clinic shows similar results, with $85 \%$ (35 out of 41 patients) of diaphragmatic resections being performed in patients with recurrent disease (7).

As is also highlighted by other working groups, the incidence of pleural effusion is higher when HIPEC perfusion is administered (34). Small diaphragmatic lesions might remain unnoticed without HIPEC. However, with HIPEC, drainage-worthy pleural effusion usually occurs. Moreover, pleural effusion occurs more frequently after fullthickness diaphragmatic resection than after diaphragmatic stripping $(35,36)$. Furthermore, when prophylactic chest tube insertion after diaphragmatic resection is omitted, approximately $50 \%$ of the patients develop pleural effusion (37). Overall, the frequency of postoperative thoracocentesis and chest tube insertion after diaphragmatic surgery in patients with ovarian cancer ranges from $14 \%$ to $42.5 \%$ (30, $31,38-40)$. According to literature data, chest drainage establishment during surgery ranges from 0 to $65 \%$ (36). In a recently published study, in which intraoperative chest tube insertion represented an integral part of diaphragmatic surgery during CRS in patients with ovarian cancer, no cases of pleural effusion or pneumothorax were detected. Owing to this, routine placement of chest drains is suggested to reduce the incidence of adverse respiratory events (41).

Since multiple organ resection takes place in the entire abdominal cavity during CRS, a certain degree of increased morbidity can be expected. The mortality rate was $3 \%$ in our patient cohort, most likely due to very high tumor burden, previous surgery, and concomitant diseases. The mortality rate reported by the same center was $2.1 \%$ in almost 500 consecutive patients, and $2.3 \%$ by the German national HIPEC registry after CRS and HIPEC, respectively $(42,43)$.

The main aim of the present study was the description of extraperitoneal peritonectomy in the upper right abdominal quadrant in patients with ovarian carcinoma as compared to the traditional approach. After the analysis of the data, the conclusion can be drawn that procedure-specific postoperative outcomes of this technique are substantially comparable to the transperitoneal approach in diaphragmatic surgery while implying several benefits as described above. Nevertheless, it has to be considered that at our tertiary reference center deals mainly with patients with locally advanced disease. Therefore, the procedures undertaken are complex and may be associated with greater morbidity.

Rodriguez et al. reported tumorous tissue in the diaphragmatic region to be a frequent cause of postoperative residual disease in advanced-stage ovarian cancer (13), which highlights the need for surgeons to be comfortable with operating in the right upper abdominal region in patients with ovarian cancer. For this reason, we advocate cytoreductive surgical management of the upper abdomen in the setting of a multidisciplinary team consisting of a gynecological oncologist and a surgical oncologist who form an ovarian team because this scenario might deliver maximal benefit to our patients. This has also been recommended by other groups (43) and demonstrated impressively by the Memorial Sloan Kettering Cancer Center group and their ovarian team (44). The potential advantages of an ovarian team include parietal peritonectomy being less time-consuming, no contamination of the pleural cavity occurs, and improved control over any incidental bleeding from either the IVC or the liver veins, thus, blood loss can be reduced. Furthermore, a higher rate of complete cytoreduction of the upper abdomen can be achieved including Glissonectomy with simplified peritonectomy of the hepatoduodenal, gastrohepatic ligament including the ligamentum venosum, the subhepatic vena cava, and the floor of the omental bursa.

As limitations, we mention the retrospective nature of our study and the inclusion of not only primary but also recurrent ovarian cancer cases, producing a heterogeneous group. The main strength of our work is that we have shown that diaphragm-related tumor tissue was always retrievable through the extraperitoneal approach, leading to complete tumor reduction in the right upper abdomen, even though our patients had a pronounced tumor burden.

\section{Conclusion}

Commonly, the right upper abdomen and right hemidiaphragm constitute the main sites of manifestation of peritoneal carcinomatosis in primary and recurrent ovarian cancer, making peritoneal stripping or full-thickness resection of the diaphragm inevitable to achieve complete cytoreduction. In the published data for gynecological oncology trials, the procedure of complete mobilization of the liver with subsequent parietal peritonectomy is mainly described. However, extraperitoneal upper quadrant peritonectomy, which has been successfully applied for one and a half decades, is a feasible procedure and an effective method to treat peritoneal carcinomatosis of the diaphragm. It provides several advantages compared to the conventional transperitoneal approach, enables a high rate of complete cytoreduction, with simplified technical performance. In particular, the preparation respects the embryological anatomical layers under constant visualization of the essential structures. However, both techniques are indicated depending on disease spread and should be available at centers for ovarian cancer surgery. Nonetheless, adequate surgical training is of paramount importance. In skilled hands, morbidity is comparable to that under the conventional approach. Expanding the surgical armamentarium with the extraperitoneal 
approach may contribute to achieving complete tumor resection in patients with ovarian cancer.

\section{Conflicts of Interest}

None of the Authors declared any potential conflict of interest with respect to the topic of the work presented here, however one author: Philipp Harter had received fees and honoraria for activities outside and without any relation to the presented work. Philipp Harter reports grants and contracts from Astra Zeneca, Roche, GSK, Genmab, DFG, European Union, DKH, Immunogen; consulting fees from Astra Zeneca, Roche, GSK, Clovis, Immunogen, MSD/Merck; honoraria for lectures and presentations from Astra Zeneca, GSK, Roche, Sotio, Stryker, Zai Lab, MSD and Clovis outside the submitted work.

\section{Authors' Contributions}

MA: Conceptualization, data collection, wrote the article; HL: wrote the article, performed the surgical procedures; $\mathrm{SH}$ : data collection and analysis, performed the surgical procedures; $\mathrm{PH}$ : contributed data; PP: Conceptualization, contributed data, performed the surgical procedures, reviewed the final version of the article. All Authors read and approved the final version of the article.

\section{References}

1 Matulonis U, Sood A, Fallowfield L, Howitt B, Sehouli J and Karlan B: Ovarian cancer. Nature Reviews Disease Primers 2(1): 16061, 2020. DOI: $10.1038 /$ nrdp.2016.61

2 Bristow RE, Tomacruz RS, Armstrong DK, Trimble EL and Montz FJ: Survival effect of maximal cytoreductive surgery for advanced ovarian carcinoma during the platinum era: a metaanalysis. J Clin Oncol 20(5): 1248-1259, 2002. PMID: 11870167. DOI: 10.1200/JCO.2002.20.5.1248

3 du Bois A, Reuss A, Pujade-Lauraine E, Harter P, Ray-Coquard I and Pfisterer J: Role of surgical outcome as prognostic factor in advanced epithelial ovarian cancer: a combined exploratory analysis of 3 prospectively randomized phase 3 multicenter trials: by the Arbeitsgemeinschaft Gynaekologische Onkologie Studiengruppe Ovarialkarzinom (AGO-OVAR) and the Groupe d'Investigateurs Nationaux Pour les Etudes des Cancers de l'Ovaire (GINECO). Cancer 115(6): 1234-1244, 2009. PMID: 19189349. DOI: $10.1002 /$ cncr.24149

4 Chi DS, Eisenhauer EL, Lang J, Huh J, Haddad L, Abu-Rustum NR, Sonoda Y, Levine DA, Hensley M and Barakat RR: What is the optimal goal of primary cytoreductive surgery for bulky stage IIIC epithelial ovarian carcinoma (EOC)? Gynecol Oncol 103(2): 559564, 2006. PMID: 16714056. DOI: 10.1016/j.ygyno.2006.03.051

5 Kehoe SM, Eisenhauer EL and Chi DS: Upper abdominal surgical procedures: liver mobilization and diaphragm peritonectomy/resection, splenectomy, and distal pancreatectomy. Gynecol Oncol 111(2 Suppl): S51-S55, 2008. PMID: 18801559. DOI: 10.1016/j.ygyno.2008.07.053

6 Zivanovic O, Eisenhauer EL, Zhou Q, Iasonos A, Sabbatini P, Sonoda Y, Abu-Rustum NR, Barakat RR and Chi DS: The impact of bulky upper abdominal disease cephalad to the greater omentum on surgical outcome for stage IIIC epithelial ovarian, fallopian tube, and primary peritoneal cancer. Gynecol Oncol 108(2): 287292, 2008. PMID: 17996927. DOI: 10.1016/j.ygyno.2007.10.001
7 Cliby W, Dowdy S, Feitoza SS, Gostout BS and Podratz KC: Diaphragm resection for ovarian cancer: technique and shortterm complications. Gynecol Oncol 94(3): 655-660, 2004. PMID: 15350355. DOI: 10.1016/j.ygyno.2004.04.032

8 Tan DS, Agarwal R and Kaye SB: Mechanisms of transcoelomic metastasis in ovarian cancer. Lancet Oncol 7(11): 925-934, 2006. PMID: 17081918. DOI: 10.1016/S1470-2045(06)70939-1

9 Shih KK and Chi DS: Maximal cytoreductive effort in epithelial ovarian cancer surgery. J Gynecol Oncol 21(2): 75-80, 2010. PMID: 20613895. DOI: 10.3802/jgo.2010.21.2.75

10 Aletti GD, Dowdy SC, Podratz KC and Cliby WA: Surgical treatment of diaphragm disease correlates with improved survival in optimally debulked advanced stage ovarian cancer. Gynecol Oncol 100(2): 283-287, 2006. PMID: 16182350. DOI: 10.1016/j.ygyno.2005.08.027

11 Eisenkop SM and Spirtos NM: Procedures required to accomplish complete cytoreduction of ovarian cancer: is there a correlation with "biological aggressiveness" and survival? Gynecol Oncol 82(3): 435-441, 2001. PMID: 11520137. DOI: 10.1006/gyno.2001.6313

12 Bommert M, Harter P, Heitz F and du Bois A: When should surgery be used for recurrent ovarian carcinoma? Clin Oncol (R Coll Radiol) 30(8): 493-497, 2018. PMID: 29743148. DOI: 10.1016/j.clon.2018.04.006

13 Rodriguez N, Miller A, Richard SD, Rungruang B, Hamilton CA, Bookman MA, Maxwell GL, Horowitz NS and Krivak TC: Upper abdominal procedures in advanced stage ovarian or primary peritoneal carcinoma patients with minimal or no gross residual disease: an analysis of Gynecologic Oncology Group (GOG) 182. Gynecol Oncol 130(3): 487-492, 2013. PMID: 23791702. DOI: 10.1016/j.ygyno.2013.06.017

14 Mehta SS, Bhatt A and Glehen O: Cytoreductive surgery and peritonectomy procedures. Indian J Surg Oncol 7(2): 139-151, 2016. PMID: 27065704. DOI: 10.1007/s13193-016-0505-5

15 Deraco M, Baratti D, Kusamura S, Laterza B and Balestra MR: Surgical technique of parietal and visceral peritonectomy for peritoneal surface malignancies. J Surg Oncol 100(4): 321-328, 2009. PMID: 19697439. DOI: 10.1002/jso.21388

16 Sugarbaker PH and Chang D: Results of treatment of 385 patients with peritoneal surface spread of appendiceal malignancy. Ann Surg Oncol 6(8): 727-731, 1999. PMID: 10622499. DOI: $10.1007 / \mathrm{s} 10434-999-0727-7$

17 Bristow RE and Montz FJ: Complete surgical cytoreduction of advanced ovarian carcinoma using the argon beam coagulator. Gynecol Oncol 83(1): 39-48, 2001. PMID: 11585412. DOI: 10.1006/gyno.2001.6344

18 Elattar A, Bryant A, Winter-Roach BA, Hatem M and Naik R: Optimal primary surgical treatment for advanced epithelial ovarian cancer. Cochrane Database Syst Rev (8): CD007565, 2011. PMID: 21833960. DOI: 10.1002/14651858.CD007565.pub2

19 Eisenhauer EL and Chi DS: Liver mobilization and diaphragm peritonectomy/resection. Gynecol Oncol 104(2 Suppl 1): 25-28, 2007. PMID: 17166568. DOI: 10.1016/j.ygyno.2006.10.037

20 Bristow RE, Karlan BY and Chi DS: Surgery for ovarian cancer. Third Edition. CRC Press, Taylor \& Francis Group, 2015.

21 Grimm C, Harter P, Heitz F and du Bois A: The sandwich technique of diaphragmatic stripping or full-thickness resection for advanced ovarian cancer: how to keep it short and simple. Int J Gynecol Cancer 25(1): 131-134, 2015. PMID: 25347091. DOI: $10.1097 / \mathrm{IGC} .0000000000000309$ 
22 Pathiraja PN, Garruto-Campanile R and Tozzi R: Diaphragmatic peritonectomy versus full thickness diaphragmatic resection and pleurectomy during cytoreduction in patients with ovarian cancer. Int J Surg Oncol 2013: 876150, 2013. PMID: 24455228. DOI: $10.1155 / 2013 / 876150$

23 Sugarbaker PH: Peritonectomy procedures. Ann Surg 221(1): 2942, 1995. PMID: 7826158. DOI: 10.1097/00000658-19950100000004

24 Sugarbaker PH: Peritonectomy procedures. Cancer Treat Res 134: 247-264, 2007. PMID: 17633058. DOI: 10.1007/978-0387-48993-3_15

25 Sugarbaker PH: Cytoreductive surgery and perioperative chemotherapy for peritoneal surface malignancy. Textbook and Video Atlas. Woodbury: CineMed Publishing Inc, 2013.

26 Hudson $\mathrm{CN}$ : A radical operation for fixed ovarian tumours. J Obstet Gynaecol Br Commonw 75(11): 1155-1160, 1968. PMID: 5726929. DOI: 10.1111/j.1471-0528.1968.tb02901.x

27 Hudson C and Chir M: Surgical treatment of ovarian cancer. Gynecologic Oncology 1(4): 370-378, 2019. DOI: 10.1016/ 0090-8258(73)90029-2

28 Montz FJ, Schlaerth JB and Berek JS: Resection of diaphragmatic peritoneum and muscle: role in cytoreductive surgery for ovarian cancer. Gynecol Oncol 35(3): 338-340, 1989. PMID: 2599468. DOI: 10.1016/0090-8258(89)90074-7

29 Chi DS, Franklin CC, Levine DA, Akselrod F, Sabbatini P, Jarnagin WR, DeMatteo R, Poynor EA, Abu-Rustum NR and Barakat RR: Improved optimal cytoreduction rates for stages IIIC and IV epithelial ovarian, fallopian tube, and primary peritoneal cancer: a change in surgical approach. Gynecol Oncol 94(3): 650654, 2004. PMID: 15350354. DOI: 10.1016/j.ygyno.2004.01.029

30 Chi DS, Zivanovic O, Levinson KL, Kolev V, Huh J, Dottino J, Gardner GJ, Leitao MM Jr, Levine DA, Sonoda Y, Abu-Rustum NR, Brown CL and Barakat RR: The incidence of major complications after the performance of extensive upper abdominal surgical procedures during primary cytoreduction of advanced ovarian, tubal, and peritoneal carcinomas. Gynecol Oncol 119(1): 38-42, 2010. PMID: 20609464. DOI: 10.1016/j.ygyno.2010.05.031

31 Chéreau E, Rouzier R, Gouy S, Ferron G, Narducci F, Bergzoll C, Huchon C, Lécuru F, Pomel C, Daraï E, Leblanc E, Querleu $\mathrm{D}$ and Morice P: Morbidity of diaphragmatic surgery for advanced ovarian cancer: retrospective study of 148 cases. Eur J Surg Oncol 37(2): 175-180, 2011. PMID: 21093204. DOI: 10.1016/j.ejso.2010.10.004

32 Ye S, He T, Liang S, Chen X, Wu X, Yang H and Xiang L: Diaphragmatic surgery and related complications in primary cytoreduction for advanced ovarian, tubal, and peritoneal carcinoma. BMC Cancer 17(1): 317, 2017. PMID: 28476108 DOI: 10.1186/s12885-017-3311-8

33 Zapardiel I, Peiretti M, Zanagnolo V, Biffi R, Bocciolone L, Landoni F, Aletti G, Colombo N and Maggioni A: Diaphragmatic surgery during primary cytoreduction for advanced ovarian cancer: peritoneal stripping versus diaphragmatic resection. Int J Gynecol Cancer 21(9): 1698-1703, 2011. PMID: 22080893. DOI: 10.1097/IGC.0b013e31822f65c3

34 Lim MC, Kang S, Choi J, Song YJ, Park S, Seo SS and Park SY: Hyperthermic intraperitoneal chemotherapy after extensive cytoreductive surgery in patients with primary advanced epithelial ovarian cancer: interim analysis of a phase II study. Ann Surg Oncol 16(4): 993-1000, 2009. PMID: 19169758. DOI: 10.1245/s10434-008-0299-y
35 Soleymani Majd H, Ferrari F, Manek S, Gubbala K, Campanile RG, Hardern K and Tozzi R: Diaphragmatic peritonectomy vs. full thickness resection with pleurectomy during VisceralPeritoneal Debulking (VPD) in 100 consecutive patients with stage IIIC-IV ovarian cancer: A surgical-histological analysis. Gynecol Oncol 140(3): 430-435, 2016. PMID: 26691220. DOI: 10.1016/j.ygyno.2015.12.004

36 Papadia A and Morotti M: Diaphragmatic surgery during cytoreduction for primary or recurrent epithelial ovarian cancer: a review of the literature. Arch Gynecol Obstet 287(4): 733-741, 2013. PMID: 23341061. DOI: 10.1007/s00404-013-2715-1

37 Bashir S, Gerardi MA, Giuntoli RL 2nd, Montes TP and Bristow RE: Surgical technique of diaphragm full-thickness resection and trans-diaphragmatic decompression of pneumothorax during cytoreductive surgery for ovarian cancer. Gynecol Oncol 119(2): 255-258, 2010. PMID: 20739051. DOI: 10.1016/j.ygyno. 2010.07.017

38 Fanfani F, Fagotti A, Gallotta V, Ercoli A, Pacelli F, Costantini B, Vizzielli G, Margariti PA, Garganese G and Scambia G: Upper abdominal surgery in advanced and recurrent ovarian cancer: role of diaphragmatic surgery. Gynecol Oncol 116(3): 497-501, 2010. PMID: 20004958. DOI: 10.1016/j.ygyno. 2009.11.023

39 Bacalbasa N, Balescu I, Diaconu C, Iliescu L, Filipescu A, Pop C, Dima S, Vilcu M and Brezean I: Right upper abdominal resections in advanced stage ovarian cancer. In Vivo 34(3): 14871492, 2020. PMID: 32354951. DOI: 10.21873/invivo.11934

40 Durmuş Y, Karalok A, Duru Çöteli SA, Boran N, Ünsal M, Boyraz $G$ and Turan T: Pulmonary morbidity related to diaphragm surgery performed for gynecological cancers. Turk J Obstet Gynecol 17(4): 292-299, 2020. PMID: 33343976. DOI: 10.4274/tjod.galenos.2020.54781

41 Carboni F, Federici O, Zazza S, Sperduti I and Valle M: Feasibility of diaphragmatic interventions in cytoreductive surgery with hyperthermic intraperitoneal chemotherapy for peritoneal carcinomatosis: A 20-year experience. Eur J Surg Oncol 47(1): 143-148, 2021. PMID: 32888734. DOI: 10.1016/ j.ejso.2020.08.016

42 Blaj S, Nedelcut S, Mayr M, Leebmann H, Leucuta D, Glockzin $\mathrm{G}$ and Piso P: Re-operations for early postoperative complications after CRS and HIPEC: indication, timing, procedure, and outcome. Langenbecks Arch Surg 404(5): 541-546, 2019. PMID: 31352505. DOI: $10.1007 / \mathrm{s} 00423-019-01808-8$

43 Piso P, Nedelcut SD, Rau B, Königsrainer A, Glockzin G, Ströhlein MA, Hörbelt R and Pelz J: Morbidity and mortality following cytoreductive surgery and hyperthermic intraperitoneal chemotherapy: Data from the DGAV StuDoQ registry with 2149 consecutive patients. Ann Surg Oncol 26(1): 148-154, 2019. PMID: 30456672. DOI: 10.1245/s10434-018-6992-6

44 Zivanovic O, Aldini A, Carlson JW and Chi DS: Advanced cytoreductive surgery: American perspective. Gynecol Oncol 114(2 Suppl): S3-S9, 2009. PMID: 19573703. DOI: 10.1016/ j.ygyno.2008.11.033 